\title{
Development of a neutron time-of-flight source at the ELBE accelerator
}

\author{
J. Klug ${ }^{*}, 1$, E. Altstadt' ${ }^{2}$, C. Beckert ${ }^{2}$, R. Beyer ${ }^{1}$, H. Freiesleben ${ }^{3}$, V. Galindo ${ }^{2}$, \\ M. Greschner ${ }^{3}$, E. Grosse ${ }^{1,3}$, A. R. Junghans ${ }^{1}$, D. Légrády ${ }^{2}$, B. Naumann ${ }^{4}$, \\ K. Noack ${ }^{2}$, K.D. Schilling ${ }^{1}$, R. Schlenk ${ }^{5}$, S. Schneider ${ }^{5}$, K. Seidel ${ }^{3}$, A. Wagner ${ }^{1}$, \\ F.-P. Weiss ${ }^{2,6}$ \\ ${ }^{I}$ Institut für Kern- und Hadronenphysik, Forschungszentrum Rossendorf, Dresden, Germany \\ ${ }^{2}$ Institut für Sicherheitsforschung, Forschungszentrum Rossendorf, Dresden, Germany \\ ${ }^{3}$ Institut für Kern- und Teilchenphysik, Technische Universität Dresden, Germany \\ ${ }^{4}$ Abteilung Sicherheit, Strahlenschutz, Forschungszentrum Rossendorf, Dresden, Germany \\ ${ }^{5}$ Abteilung Mechanische Entwicklung, Forschungszentrum Rossendorf, Dresden, Germany \\ ${ }^{6}$ Institut für Energietechnik, Technische Universität Dresden, Germany \\ E-mail: j.klug@fz-rossendorf. de
}

The ELBE electron beam at Forschungszentrum Rossendorf, Dresden, with energies up to 40 $\mathrm{MeV}$, will be used to produce intense neutron beams. The neutron radiator consists of a liquid lead circuit where bremsstrahlung photons created by the electrons produce neutrons in $(\gamma, n)$ reactions. The short beam pulses $(5 \mathrm{ps})$ provide the basis for an excellent time resolution for neutron time-of-flight experiments, giving an energy resolution of about $1 \%$ with a flight path of $4 \mathrm{~m}$. The neutron beam is shaped by a $2.4 \mathrm{~m}$ long collimator. The usable energy range is from $50 \mathrm{keV}$ up to $10 \mathrm{MeV}$ when ELBE delivers a beam repetition rate of $0.5 \mathrm{MHz}$. In this energy interval, there is a need for neutron cross section measurements relevant for the transmutation of minor actinides in nuclear waste, as well as for applications to fission and fusion reactors. The neutron flux at the sample position is $10^{7} \mathrm{~cm}^{-2} \mathrm{~s}^{-1}$.

Different detector types are being developed. For neutron capture $\gamma$ rays, a $\mathrm{BaF}_{2}$ scintillation detector array of 42 crystals is being constructed, whereas for neutron detection, an up to $1 \mathrm{~m}^{2}$ large plastic scintillator wall consisting of $1 \mathrm{~cm}$ thick scintillation panels is being built.

International Workshop on Fast Neutron Detectors and Applications University of Cape Town, South Africa

April 3-6, 2006

\section{${ }^{*}$ Speaker}




\section{Liquid-lead neutron radiator}

A very compact neutron time-of-flight system under development at the superconducting electron linear accelerator ELBE $^{*}$ at Forschungszentrum Rossendorf in Dresden will use the electron beam to produce neutrons in a liquid-lead neutron radiator. The electron beam micropulse duration is $5 \mathrm{ps}$ and the energy can be varied up to $40 \mathrm{MeV}$. A superconducting RF photo electron injector (SRF gun) is being developed, aiming at an electron beam current of $1 \mathrm{~mA}$ at a repetition rate of $0.5 \mathrm{MHz}$ [1]. The measurable neutron energy range is $50 \mathrm{keV}$ to $10 \mathrm{MeV}$.

When operating the liquid-lead radiator, the electron beam passes a beryllium window mounted on a stainless steel vacuum chamber and hits the radiator, consisting of a molybdenum channel confining the liquid lead (see figure 1). The electrons create bremsstrahlung photons which in secondary processes release neutrons. These leave the radiator almost isotropically, while the electrons and photons

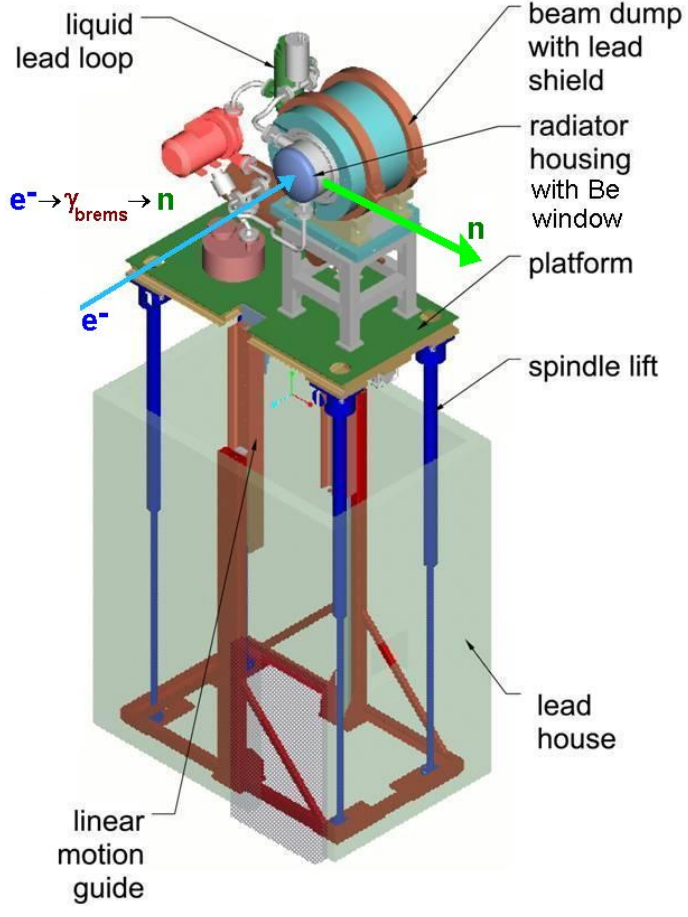

Figure 1. 3D rendering of the pulsed neutron radiator in the neutron production cave of the ELBE building. The liquid-lead loop with the radiator and the beam dump can be lowered into a lead house when not operated. are forward-peaked and stopped in a lead-shielded, water-cooled aluminium beam dump. By using the neutrons emitted perpendicularly from the electron beam, a high suppression of the electrons and photons is obtained. A collimator creates a well-defined neutron beam that enters the experimental site in the adjacent room. Apart from the molybdenum channel in the radiator, the liquid lead is transported in an insulated and electrically heatable stainless-steel tube circuit and cooled through a heat exchanger using an InGaSn eutecticum as an intermediate heat transfer fluid.

With a radiator volume of approximately $1 \mathrm{~cm}^{3}$ the time interval of beam passage and photoneutron production is kept in the sub-nanosecond range, and the scattering inside the radiator as well as the background of thermal neutrons is minimized. At the same time, this volume is large enough to obtain a reasonable conversion efficiency. Liquid lead was chosen as radiator material since the thermal load deposited by the electron beam (up to $25 \mathrm{~kW}$ ) is too high to be dissipated from a solid target of such small size by gas cooling and heat radiation. Cooling with water would be unfavorable due to neutron scattering and moderation.

Monte Carlo simulations were performed using MCNP4C3 [2] to characterise neutron and photon intensities, and time and energy distributions. The main parameters determining the

\footnotetext{
${ }^{*}$ Electron Linear accelerator with high Brilliance and low Emittance
} 
neutron intensity at the sample position are the beam current, the energy of the electron beam, the length of the neutron flight path, and the radiator dimensions. For electrons with energy $E_{\mathrm{e}}=30 \mathrm{MeV}$ and a beam current of $I_{\mathrm{e}}=1 \mathrm{~mA}$ the simulations predict a neutron source strength of $2 \cdot 10^{13} \mathrm{~s}^{-1}$ from the radiator and a neutron flux density of $1 \cdot 10^{7} \mathrm{~cm}^{-2} \mathrm{~s}^{-1}$ at the measuring position $3.9 \mathrm{~m}$ from the radiator (behind the collimator).

Figure 2 shows the energy distribution of the significant contributions to the neutron flux density at the measuring position, with $E_{\mathrm{e}}=30 \mathrm{MeV}$ and $I_{\mathrm{e}}=1 \mathrm{~mA}$. The total distribution is represented by black diamonds. Almost $92 \%$ stem directly from the lead in the radiator (grey squares), while about $8 \%$ of the neutrons were scattered or created in the molybdenum channel (red circles). A very small fraction $(<0.1 \%$ ) was scattered in the steel housing accommodating the radiator (green triangles).

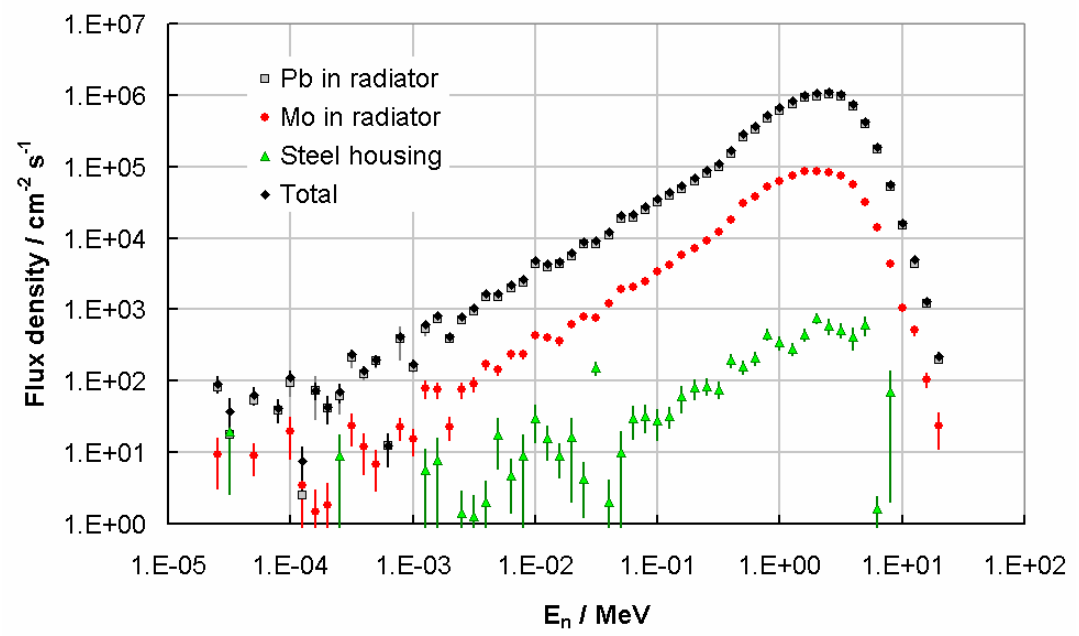

Figure 2. Contributions to the neutron flux density at the measuring position $3.9 \mathrm{~m}$ from the radiator (for each neutron energy bin). Electron energy $E_{\mathrm{e}}=30$ $\mathrm{MeV}$, beam current $I_{\mathrm{e}}=$ $1 \mathrm{~mA}$.

\section{Collimator}

The collimator must eliminate neutrons and photons scattered or produced in the collimator, and create a spatially well-defined beam of unscattered neutrons with a sharp edge and a minimal background of both neutrons and photons outside the beam. In addition, the component of slow neutrons coming directly from the radiator, overlapping into the next beam pulse and creating measurement ambiguities, has to be minimized using a filter in the beam.

Figure 3 shows four examples of investigated collimator compositions placed in the wall consisting of $1.2 \mathrm{~m}$ concrete and $1.2 \mathrm{~m}$ of heavy concrete (seen from left to right). Grey collimator insertions are made from lead and yellow ones from borated polyethylene (CHB), while uncoloured regions do not contain any material. The neutrons travel from left to right.

All collimators shape a neutron beam with a sharp edge indicated by the intensity drop outside the hole radius of $1.5 \mathrm{~cm}$, and at larger distances the intensity is 4-5 orders of magnitude less than in the beam. The collimator compositions not fully filled with material have the lowest background outside the beam, and the best solution is with a conical hole focussing on the radiator. 


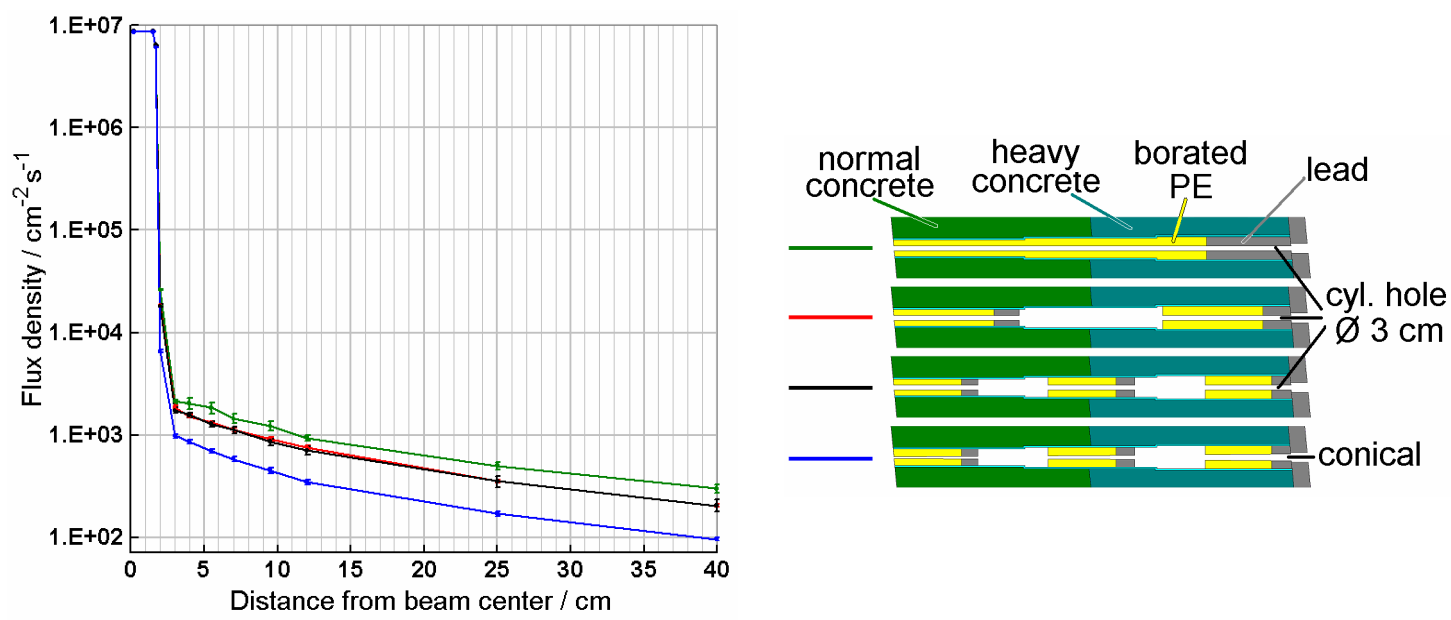

Figure 3. Neutron flux densities after four different collimator types (3.9 $\mathrm{m}$ from the neutron radiator), at increasing distance from the beam center $\left(E_{\mathrm{e}}=30 \mathrm{MeV}, I_{\mathrm{e}}=1 \mathrm{~mA}\right)$. The coloured lines indicate the neutron beam profile behind the respective collimator shown in the right part of the figure.

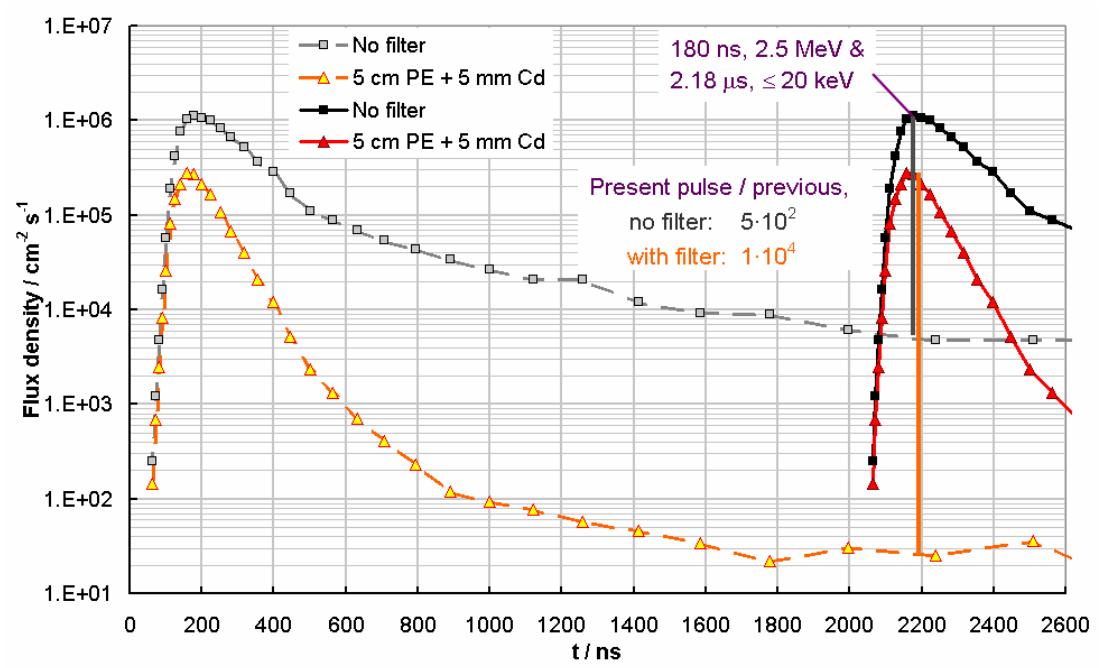

Figure 4. Neutron flux time distributions of two consecutive beam pulses, using an absorber for low-energy neutrons (triangles), and no absorber (squares), respectively.

An unambiguous assignment of energy to a neutron with a certain flight time requires that slow neutrons from previous beam pulses are eliminated with a filter. Figure 4 shows the time distribution of two consecutive pulses with a repetition rate of $500 \mathrm{kHz}$. The upper graphs (grey and black) represent the situation without a filter, while for the lower ones (yellow and red) a filter of $5 \mathrm{~cm}$ polyethylene and $5 \mathrm{~mm} \mathrm{Cd}$ was used. The cost of using the absorber is a decrease in peak intensity by a factor of 5, but the relative background reduction is considerable. If, e.g., a $2.5 \mathrm{MeV}$ neutron arriving $180 \mathrm{~ns}$ after leaving the radiator is measured in the latter pulse, the "background" flux from the previous pulse lies a factor 500 below, with energies up to $20 \mathrm{keV}$. With the filter in place, this ratio changes to $10^{4}$.

The correlation between neutron kinetic energy $\left(E_{\mathrm{n}}\right)$ and time of flight (ToF) is shown in figure 5, focusing on the region around the flux density maximum. The main ridge in the 
spectrum is from neutrons with a correct energy to ToF correlation. Neutrons that have lost their undisturbed correlation between $E_{\mathrm{n}}$ and ToF form a tail to the main ridge. They are supressed by two to three orders of magnitude, and constitute only $4 \%$ of all events. The low intensity ridge approximately $20 \mathrm{~ns}$ above the distribution of unscattered neutrons is due to scattering in the stainless steel housing around the radiator. The inset shows a horizontal cut through the spectrum at $230 \mathrm{~ns}$. The energy width (FWHM) of the peak for neutrons with this ToF is $5 \mathrm{keV}$, which corresponds to an energy resolution less than $1 \%$ at $E_{\mathrm{n}}=1.5 \mathrm{MeV}$. In addition to this, the time resolution of the ToF detector has to be included to determine the total experimental energy resolution.

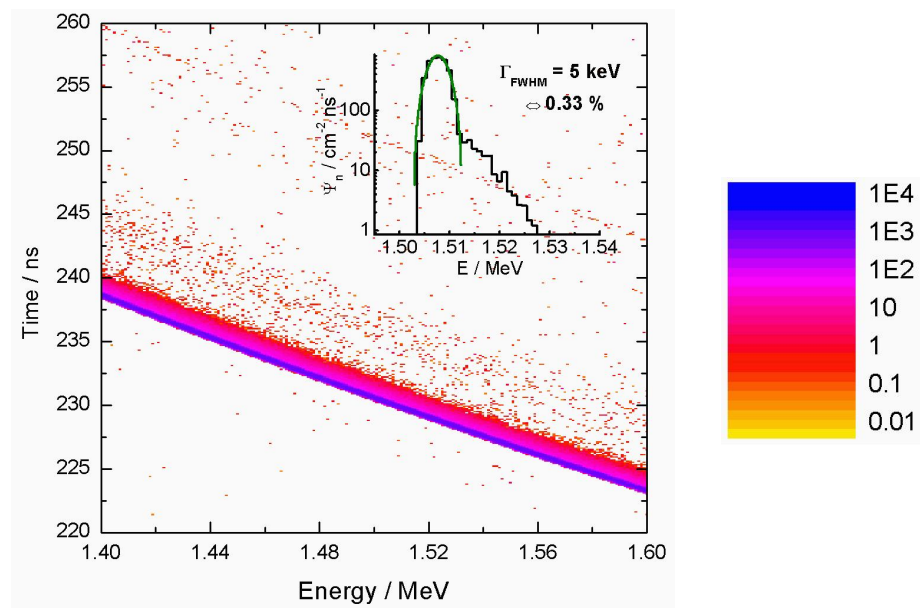

Figure 5. The correlation between neutron time-of-flight and energy is preserved for $96 \%$ of the neutrons, which form a narrow, dark band in a ToF-vs- $E_{\mathrm{n}}$ scatter plot. The inset shows a horizontal cut of the correlation at $230 \mathrm{~ns}$, giving an energy resolution at FWHM of less than $1 \%$ at $E_{\mathrm{n}}=1.5 \mathrm{MeV}$.

\section{Detector development}

For measurements of neutron capture $\gamma$-rays, a $\mathrm{BaF}_{2}$ scintillation detector array of 42 crystals covering $80 \%$ of the total solid angle is being built. The crystals are $19 \mathrm{~cm}$ long and have a hexagonal cross section with an inner diameter of $53 \mathrm{~mm}$. They are read out by fast Hamamatsu R2059 PM tubes, which are UV sensitive to be able to measure both the slow and the fast component of the $\mathrm{BaF}_{2}$ scintillation light. Thereby pulse shape discrimination (PSD) can be utilised to separate photon signals from intrinsic $\alpha$-particle background. The time resolution attained with a ${ }^{60} \mathrm{Co} \gamma$-source is typically $650 \mathrm{ps}$ (FWHM). The readout will be performed with dedicated ADC/TAC modules [3] that allow simultaneous measurement of timing and energy signals including PSD in VMEbus standard.

For neutron detection, a plastic scintillator wall is being constructed from $1 \mathrm{~m}$ long scintillator stripes read out at both ends, which allows for position determination. A position resolution of $5 \mathrm{~cm}$ was established using electrons from a collimated ${ }^{90} \mathrm{Sr}$ source.

It has been shown that it is experimentally possible to reach a threshold of $5-10 \mathrm{keV}$ for the plastic scintillator detectors by triggering just below the single-electron peak and using a coincident readout of the two PM tubes [4] (see figure 6). Therefore, simulations were done to compare these detectors below $500 \mathrm{keV}$ with lithium-glass neutron detectors (utilizing the ${ }^{6} \mathrm{Li}(\mathrm{n}, \mathrm{t}) \alpha$ reaction), often used in this low-energy region. The detector types were compared by 
means of their neutron detection efficiencies and their time smearing due to scattering in the scintillator material before detection. As an example, when $144 \mathrm{keV}$ neutrons were hitting a 25 $\mathrm{mm}$ thick lithium-glass detector, $34 \%$ of all events had a degraded timing information due to multiple scattering - see figure 7 . For an $11 \mathrm{~mm}$ thick plastic scintillator, with a threshold on the proton recoil energy of $5 \mathrm{keV}$, the share of such events was $15 \%$. The simulated detection efficiencies are also in favour of plastic scintillators (60-80\%, depending on thickness) compared to Li-glass detectors $(<5 \%)$. This can be explained with the higher number density of protons in plastic, compared to that of ${ }^{6} \mathrm{Li}$ nuclei in lithium-glass, and with the larger $\mathrm{np}$ scattering cross section. Hence, the simulations indicate that the plastic scintillators can be used also in the low-energy end of the ELBE neutron energy range. An effect that will play a significant role at these low energies is the quenching of the light output from recoil protons compared to that from electrons of the same energy. Also, the non-linear light output from recoil protons tends to decrease the detection efficiency. The absolute efficiency will be investigated experimentally at the neutron beam facility of Physikalisch-Technische Bundesanstalt (PTB) in Braunschweig, Germany.
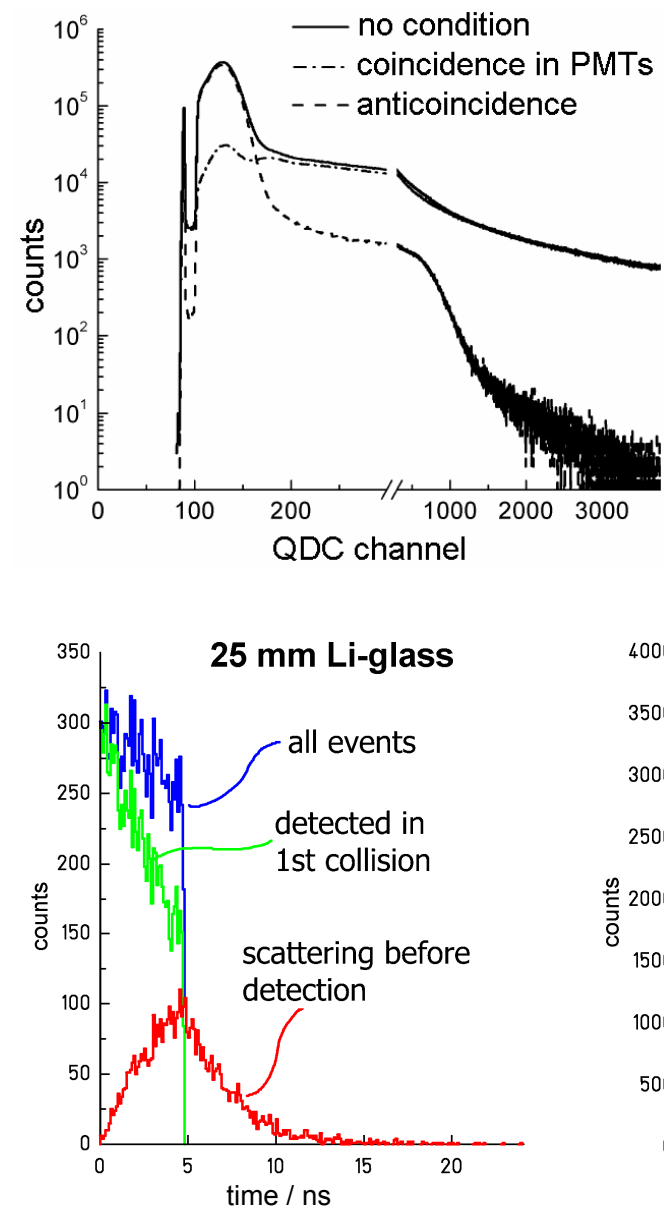

Figure 6. QDC spectrum of a plastic scintillator Hamamatsu R2059 PM tube, in coincidence and in anticoincidence (respectively) with the PM tube at the other end.

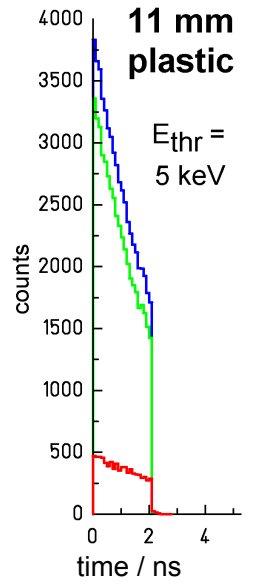

Figure 7. Simulated detector time spectra. A $25 \mathrm{~mm}$ thick lithium-glass detector (left) is compared to an $11 \mathrm{~mm}$ thick plastic scintillator detector, subject to a proton recoil energy threshold of $5 \mathrm{keV}$ (right). The neutrons are started at time $0 \mathrm{~ns}$. 


\section{References}

[1] J. Teichert, Forschungszentrum Rossendorf, private communication, 2005, and http://www.fz-rossendorf.de/pls/rois/Cms?pNid=604.

[2] J. F. Briesmeister (ed.) MCNP - a General Monte Carlo N-Particle Transport Code (Report LA13709), Los Alamos National Laboratory 2000.

[3] C. P. Drexler, U. Thöring et al., IEEE Trans. Nucl. Sci. 50 (2003) 969.

[4] R. Beyer, Diplomarbeit, http://www.db-thueringen.de/servlets/DocumentServlet?id=5298 (2005) 